[Chem. Pharm. Bull.

35(11)4405-4409(1987)

\title{
A Velocity-Gradient Flow Cell for Raman and Emission Spectroscopy of a Deoxyribonucleic Acid-Drug Solution
}

\author{
Masamichi Tsuboi, ${ }^{*, a, 1)}{\text { Teruki IKeda },{ }^{b} \text { and Heizaburo Shindo }}^{c}$ \\ Department of Chemistry, Meisei University, ${ }^{a}$ Hodokubo 2-1-1, Hino-shi, Tokyo 191, Japan, \\ Application Laboratory, Japan Spectroscopic Co., Ltd., ${ }^{b}$ 2967-5, Ishikawa-cho, \\ Hachioji-shi, Tokyo 192, Japan, and Division of Analytical Chemistry, \\ Tokyo College of Pharmacy, ${ }^{c}$ Horinouchi, Hachioji-shi, \\ Tokyo 192-03, Japan
}

(Received April 28, 1987)

\begin{abstract}
A deoxyribonucleic acid (DNA) solution was placed in the gap (1 mm) between two coaxial cylinders; the inside diameter of the outer cylinder was $12 \mathrm{~mm}$, and the outside diameter of the inner cylinder was $10 \mathrm{~mm}$. When the inside cylinder was rotated at 2500 revolutions per minute, a sufficiently large velocity gradient of the liquid flow was produced across the gap so that the DNA molecules were oriented along the flow direction. Anisotropy of the Raman scatterings of the DNA molecule was examined by passing an exciting laser beam into the gap from the bottom of the cylinder in the direction parallel to the cylinder axis. An antitumor drug, aclacinomycin $\mathrm{A}(\mathrm{HCl}$ salt), was added to the DNA solution, and the anisotropy of its emission and Raman spectra was also examined. It has been established that aclacinomycin binds to DNA with its chromophore oriented parallel to the base plane of the DNA duplex.
\end{abstract}

Keywords_aclacinomycin; DNA; Raman spectrum; emission spectrum; flow cell

In ordinary solutions, the molecules are randomly oriented. In a spectroscopic measurement on such a solution, therefore, information on the anisotropic properties of the molecules in question is obscured. It is true that a spectroscopic measurement on a single crystal has a number of advantages in this respect. However, it is often difficult to obtain crystalline samples in biological and pharmaceutical studies. In addition, what is usually relevant in such studies is information on the molecules in solution rather than in crystals, where the molecular environment may.be greatly different from that in a living system. Thus it is desirable to have molecules oriented in solution. This is known to be possible for the deoxyribonucleic acid (DNA) duplex by placing its solution in a flow with a velocity gradient of $10^{3} \mathrm{~cm} \mathrm{~s}^{-1} / \mathrm{cm}$ order of magnitude. ${ }^{2,3)}$ Wada and Kozawa, ${ }^{3)}$ for example, produced such a velocity gradient in the flow of a solution placed in the gap between two coaxial cylinders, one of which was rapidly rotated. Wada et al. examined the anisotropy of the $260 \mathrm{~nm}$ absorption of DNA, and of the $445 \mathrm{~nm}$ absorption of actinomycin D bound to DNA. ${ }^{3,4)}$ In our present study, a similar technique has been applied to Raman and fluorescence spectroscopy. This has proved to be a powerful technique for characterizing the Raman lines of DNA, and for elucidating the geometry of a DNA-drug interaction.

\section{Description of the New Cell: a Velocity-Gradient Flow Cell for Laser Raman and Emission Spectroscopy ${ }^{5)}$}

A solution container has been constructed, in which a flow of the sample solution, having a uniform velocity gradient, can be produced. It consists of two coaxial cylinders (inner and outer cylinders) whose dimensions are given in Fig. 1. As may be seen in the figure, a gap of $1 \mathrm{~mm}$ is produced when the inner cylinder is inserted into the outer cylinder along the common vertical axis. The sample solution is placed in this gap. When the inner cylinder is 


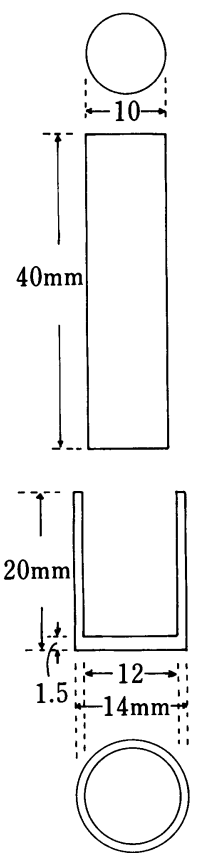

Fig. 1. Two Coaxial Glass Cylinders, Which Form the Essential Portion of the New Flow Cell

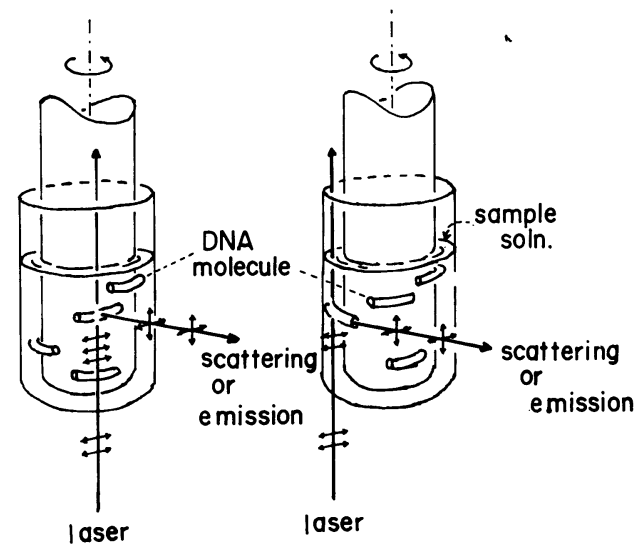

$Z$-arrangement

$X$-arrangement

Fig. 2. Two Different Scattering or Emission Geometries for the New Flow Cell

rotated at the rate of 2500 revolutions per minute $(\mathrm{rpm})$ with the outer cylinder fixed, a flow of the solution is produced along the horizontal circles. The flow velocity is zero at the outer wall, whereas it is about $1300 \mathrm{~mm} / \mathrm{s}$ at the other end of the gap (at the surface of the inner cylinder). Therefore, the velocity gradient here, which would be nearly uniform, should be $1300 \mathrm{~s}^{-1}$.

For examining the Raman and/or emission spectrum of the sample solution, the exciting laser beam is passed from the bottom vertically along the gap through the solution. The light scattered or emitted from the sample solution along the horizontal direction through the outer cylinder is collected and led to the entrance slit of a spectrometer. Two different modes of passing the laser beam were adopted: in one of them, the electric vector of the laser beam was directed along the flow of the solution ( $Z$-arrangement, see Fig. 2$)$; in the other, the laser beam was polarized along the perpendicular direction to the flow $(X$-arrangement, see Fig. 2$)$. For locating the outer and inner cylinders relative to each other and relative to the laser and spectrometer positions, it is essential to have micropositioners for both cylinders which should be operated independently of each other.

\section{Example I: Effect of Aligning DNA Molecules on the Raman Spectrum}

A sample of salmon sperm DNA was obtained from PL Biochemicals Inc., and used after being purified as follows: DNA was dissolved in $10 \mathrm{~mm}$ Tris $+0.1 \mathrm{~mm}$ EDTA (ethylenediaminetetraacetic acid) solution ( $\mathrm{pH} 7.5)$ and stirred overnight; 2 volumes of ethanol were added to precipitate DNA, which was dried in the atmosphere and then dissolved in $0.1 \mathrm{M}$ $\mathrm{NaCl}+0.1 \mathrm{~mm}$ EDTA overnight. Finally the solution was subjected to centrifugation at 20000 $\mathrm{rpm}$ for $2 \mathrm{~h}$ to remove precipitates.

This DNA solution (concentration $=0.5 \%$ ) was placed in the cell and the Raman spectrum was observed, by the use of a Jasco NR-1000 Raman spectrometer, with the $X$ - and 
$Z$-arrangements of the $488.0 \mathrm{~nm}$ beam of an NEC GLG $3200 \mathrm{Ar}$ ion laser. As may be seen in Fig. 3, the intensities of some observed Raman lines are markedly different for $X$ - and $Z$ arrangements. This fact indicates that the sample solution is anisotropic, and this is considered to be caused by alignment of the DNA molecules along the flow. For a DNA duplex with its axis along the $Z$-direction (flow direction), we can expect four different components of each Raman scattering tensor, namely $x x, x y, x z$, and $z z$ components. The optical system collecting the scattering light has no polarizer, and the spectrometer has a scrambler. Therefore, the scattering light should involve mainly the $x x$ and $x y$ components in the $X$-arrangement, and the $x z$ and $z z$ components in the $Z$-arrangement (see Fig. 2). It is understandable that the $1375 \mathrm{~cm}^{-1}$ line, assignable to the $\mathrm{CH}_{3}$ symmetric deformation vibration of the thymine residue, and the $1090 \mathrm{~cm}^{-1}$ line, assignable to the $\mathrm{PO}_{2}^{-}$symmetric stretching vibration, have relatively large $z z$ components. Thus, both of these vibrations have appreciable atomic displacements along the $z$-direction (axis of the DNA double helix), and therefore may cause large polarizability oscillation along the $z$-direction. On the other hand, the $1490,1338,1310$, and $1270 \mathrm{~cm}^{-1}$ lines, all assignable to in-plane skeletal stretching vibrations of the base residues, would have large $x x$ and $x y$ components, but only a smaller $z z$ component. This explains why these lines are weak in the spectrum with the $Z$-arrangement of the exciting laser.

\section{Example II: Effects on the Raman and Emission Spectra of Aclacinomycin Bound to DNA} Aclacinomycin A has antitumor activity, ${ }^{6}$ and is known to be bound strongly to DNA in

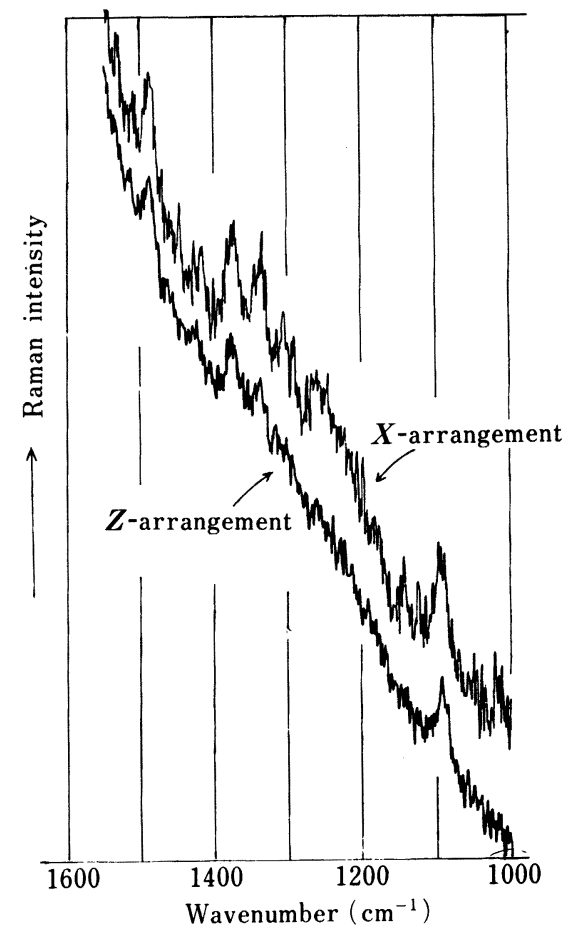

Fig. 3. Raman Spectra of Salmon Sperm DNA in $0.5 \%$ Aqueous Solution at $\mathrm{pH} 7.5$ (No Buffer), and at $20^{\circ} \mathrm{C}$

Exciting light, $488.0 \mathrm{~nm}$ beam of an $\mathrm{Ar}$ ion laser ( $100 \mathrm{~mW}$ at the sample point). Two different modes of incidence are adopted (see Fig. 2).

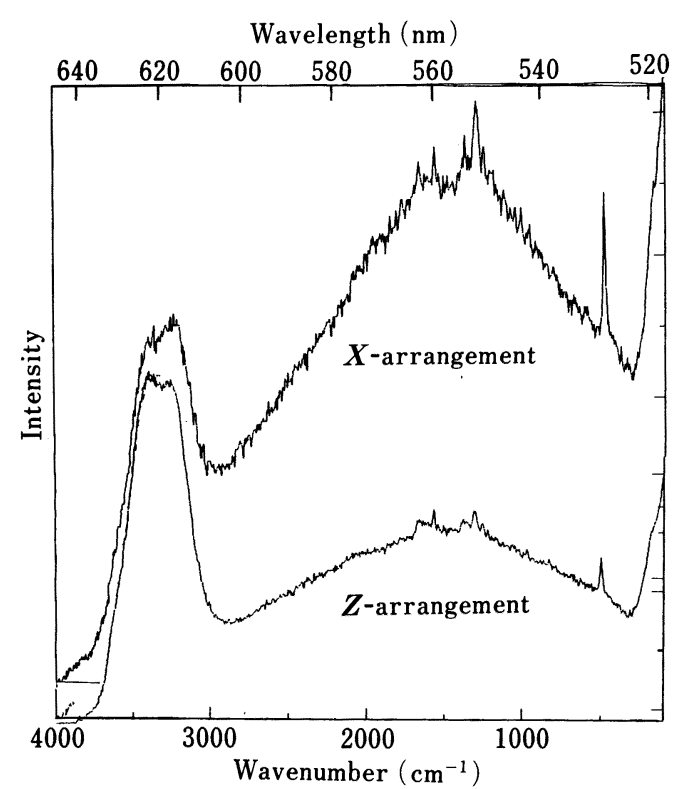

Fig. 4. Raman and Fluorescence Spectra of an Aqueous Mixture of Salmon Sperm DNA $(0.15 \mathrm{M}$ in Nucleotide) and Aclacinomycin A $\mathrm{HCl}(0.0005 \mathrm{M})$, at $\mathrm{pH} 6$ and at $20^{\circ} \mathrm{C}$

Exciting light, $514.5 \mathrm{~nm}$ beam of Ar ion laser ( $30 \mathrm{~mW}$ at the sample point). Two different modes of incidence are adopted (see Fig. 2). 
aqueous solution. ${ }^{7,8)}$ This drug is strongly fluorescent but the fluorescence is markedly quenched on binding to DNA. Is the residual fluorescence caused by the drug bound to DNA? Does this quenching enable us to observe the Raman spectrum of aclacinomycin? What is the geometry of the chromophore of this drug with respect to DNA in the complex? These questions were all answered by a spectroscopic examination by the use of the present new "velocity gradient flow cell."

Aclacinomycin $\mathrm{A} \mathrm{HCl}$ was added to the above-mentioned DNA solution, so that the final concentrations of DNA and drug were $0.15 \mathrm{M}$ and $0.0005 \mathrm{M}$, respectively. This mixture solution was placed in the cell, and the scattering and emission were examined with a Jasco NR-1100 Raman spectrometer, for the $X$ - and $Z$-arrangements of the $514.5 \mathrm{~nm}$ beam of an NEC GLG 3200 Ar ion laser. The results are shown in Fig. 4. As may be seen here, the fluorescence background is much stronger for the $X$-arrangement than for the $Z$-arrangement. This is clearly seen by using the $\mathrm{H}_{2} \mathrm{O}$ Raman band at around $3300 \mathrm{~cm}^{-1}$ (at $620 \mathrm{~nm}$ ) as an internal standard; the water molecules in the solution would be directed randomly even under the flow, and the Raman band should have equal intensities for the $X$ - and $Z$-arrangements of the laser. Thus, the transition moment of the $558 \mathrm{~nm}$ emission band of the aclacinomycin chromophore has now been shown to be directed dominantly along the $X$-direction (perpendicular to the flow, or to the axis of the aligned DNA duplex) rather than the $Z$ direction. It has been indicated, in other words, that the fluorescence in question arises from the aclacinomycin molecule bound to DNA, and that its chromophore is located parallel to the DNA base plane. This gives support to the idea that the chromophore is intercalated in the stacked base-pairs of the DNA molecule.

Superimposed on the fluorescence background just mentioned, several prominent Raman lines are evident (see Fig. 4). They are at 500, 1300, 1380, 1590, and $1700 \mathrm{~cm}^{-1}$, and are assignable to ring vibrations of the aclacinomycin chromophore. These are considered to be resonance-enhanced by the $437 \mathrm{~nm}$ absorption, whereas the Raman lines of DNA are much weaker so that they are buried in the fluorescence background. All of the observed Raman lines of aclacinomycin are stronger for the $X$-arrangement of the laser than for the $Z$ arrangement. This is understandable, because these Raman lines should be caused by in-plane vibrations of the chromophoric portion of the aclacinomycin molecule, and here every Raman scattering should have a greater $x x$ component than $z z$ component.

\section{Discussion}

The experiments described in the preceding two sections (Examples I and II) have proved that the present velocity-gradient flow cell is useful (1) in aligning the DNA molecules unidirectionally in solution, to obtain knowledge of the anisotropy of the DNA Raman lines, (2) for judging whether a given drug molecule is bound to DNA, and (3) if so, for obtaining information on the geometry of the complex. The cell would also be useful (4) for aligning any rod-shaped macromolecules or their complexes, including some viruses such as tobacco mosaic virus. This may allow us to obtain information on the relative orientations of the constituent molecules. Lastly, the cell should be useful (5) for examining in detail the dynamic behavior of macromolecules and for deriving their elastic constants in a quantitative manner. When the velocity gradient of the flow of a sample solution is continuously changed, and when the anisotropy of its emission, for example, is quantitatively determined as a function of the velocity gradient and as a function of ionic strength and other properties of the solvent, the results would represent valuable data on the dynamic properties of macromolecule or a macromolecular complex. 
advice, to Dr. Tomio Takeuchi (Institute of Microbial Chemistry) and Dr. Hiroshi Tone (Sanraku-Ocean Co., Ltd.) for the aclacinomycin A HCl sample, and to Mr. Tadashi Miyazaki (President, Japan Spectroscopic Co., Ltd.) for his interest, encouragement, and support. This work was partly supported by a grant (No. 62840014) from the Ministry of Education, Science, and Culture.

\section{References and Notes}

1) Present address: Faculty of Science and Engineering, Iwaki-Meisei University, Chuodai-Iino 5-5-1, Iwaki, Fukushima 970, Japan.

2) A. Wada, Applied Spectroscopy Reviews, 6, 1 (1972).

3) A. Wada and S. Kozawa, J. Polym. Sci., A2, 853 (1964).

4) M. Tsuboi, S. Higuchi, Y. Kyogoku, K. Matsuo, and A. Wada, Chem. Pharm. Bull., 12, 501 (1964).

5) Patent pending.

6) T. Oki, Y. Matsuzawa, A. Yoshimoto, K. Numata, I. Kitamura, S. Hori, A. Takamatsu, H. Umezawa, M. Ishizuka, H. Naganawa, H. Suda, M. Hamada, and T. Takeuchi, J. Antibiot., 28, 830 (1975).

7) H. Yamaki, H. Suzuki, T. Nishimura, and N. Tanaka, J. Antibiot., 31, 1149 (1978).

8) S. Takahashi, N. Nagashima, Y. Nishimura, and M. Tsuboi, Chem. Pharm. Bull., 34, 4494 (1986). 